\title{
Gratitud en el aula
}

\section{Gratitude in the Classroom}

José Miguel García Ramírez. Postdoctoral Fellow at Trent University

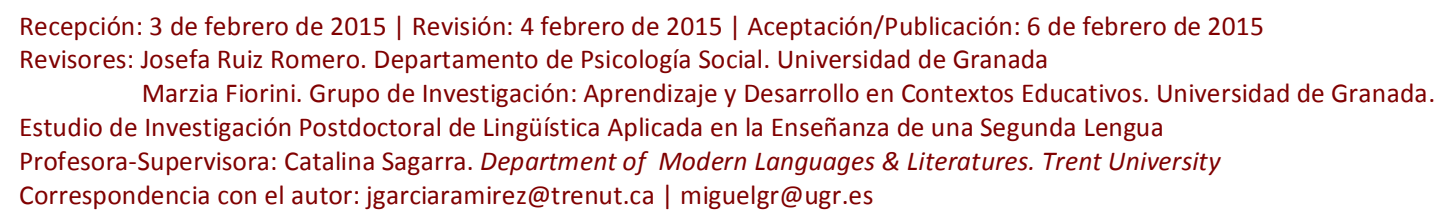

\section{Resumen}

La gratitud es una fortaleza psicológica que facilita la adquisición y construcción social del conocimiento, infiriendo intencionalidad y búsqueda del beneficio compartido como intercambio recíproco guiado por las emociones y el afecto. Con esta investigación se demuestra que la acción de mostrar gratitud influye positivamente en el rendimiento académico. Método: Estudio exploratorio realizado durante el primer semestre del curso académico 2013-14 y el primer semestre del curso académico 2014-15 en Trent University (Canadá). Ciento cuarenta y cinco estudiantes de Estudios Hispánicos fueron adscritos al Grupo Experimental o al Grupo Control. Al grupo experimental se le aplicó un programa de agradecimiento simple; para el análisis del impacto del programa fueron utilizadas dos pruebas objetivas (pre y post) de rendimiento académico. Resultados: El análisis estadístico muestra que existen diferencias significativas entre los resultados obtenidos por el Grupo Control y el Grupo Experimental, cuyos integrantes obtuvieron un mejor rendimiento que los del grupo control. Conclusiones: La gratitud mejora el rendimiento académico en el aprendizaje de las lenguas y ayuda a consolidar una educación humanística.

Palabras clave: Gratitud | Reciprocidad | Educación | Rendimiento

\begin{abstract}
Gratitude is a psychological strength that facilitates acquisition and social construction of knowledge, inferring intent and a search for shared benefit as a reciprocal exchange guided by emotions and affection. This research study shows that the act of expressing gratitude has a positive influence on academic achievement. Method: An exploratory study conducted during the first semester of the 2013-14 academic year and the first semester of the 2014-15 academic year at Trent University (Canada). The participants were 145 students of Hispanic Studies, randomly assigned to the experimental group or the control group. The experimental group received a simple appreciation program; the instruments used were two objective tests (pre and post) from the academic evaluation program. Results: The statistical analysis shows that there are significant differences between the results obtained by the control group and the experimental group. Conclusions: Gratitude improves academic performance in language learning and helps to consolidate a humanistic education.
\end{abstract}

Keywords: Gratitude | Reciprocity | Education | Performance 


\section{Introducción}

La gratitud es considerada una fortaleza psicológica relacionada con las conductas pro-sociales, emociones positivas, agrado con la propia vida, el optimismo y la percepción subjetiva de felicidad (Peterson, y Seligman, 2005; Peterson, Ruch, Beermann, Park, y Seligman, 2007); también está relacionada con el bienestar emocional, el nivel de felicidad, la satisfacción y la calidad de vida (Brülde, 2007), a pesar que en algunos estudios hayan encontrado diferencias entre gratitud y el sentido de "estar en deuda con otros" y la definan como la apreciación emitida después de recibir cualquier tipo de ayuda (Tsang, 2006; Watkins, Scheer, Ovnicek, y Kolts, 2006). No obstante, la gratitud es un estado emocional positivo que se experimenta después de recibir, desinteresadamente, algo. En este sentido la mayoría de las investigaciones asocian la gratitud con los constructos de optimismo, empatía, simpatía y satisfacción con la vida, dando lugar a una vida placentera y de compromiso pro-social (McCullough, Emmons y Tsang, 2002); también, la gratitud ayuda a percibir positivamente el contexto, reduce los efectos de la estima y refuerza la visión de autonomía personal, obteniendo satisfacción, felicidad y bienestar (Sheldon y Lyubomirsky, 2006; Wood, Froh, y Geraghty, 2010).

Según Emmons (2007) hay tres condiciones que producen gratitud: $1^{\text {a) }}$ La persona agradecida debe percibir que ha sido beneficiada con algo que tiene valor; la relación de la importancia de lo recibido con el sentido de gratitud es directamente proporcional, cuanta más importancia se le otorgue a lo recibido, más agradecimiento se muestra. $2^{a}$ ) La persona debe reconocer al agente que lo ha beneficiado; así pues, mientras más se valore a la persona que nos beneficia, mayor será también el grado de gratitud experimentado. $3^{a}$ ) Se debe percibir el beneficio como un regalo, es decir, he sido beneficiado de un modo gratuito.

Esas condiciones, de carácter cognitivo, necesitan también una respuesta emocional específica, acompañada de una emoción positiva e intensa, para poder afirmar que una persona está experimentando gratitud o agradecimiento (Emmons, y McCollough, 2003), por lo que las personas que muestran agradecimiento suelen ser más felices haciendo uso de la inteligencia emocional y social (McCullough, Tsang, y Emmons, 2004; Sheldon y Lyubomirsky, 2006).

Por lo tanto, la gratitud se puede considerar necesaria para desarrollar una enseñanza humanística; ya que su carácter cognitivo-emocional facilita la adquisición y construcción del conocimiento, mejorando la interacción y reciprocidad entre sus participantes, dentro de un contexto de interdependencia positiva y de motivación intrínseca (Emmons, y Crumpler, 2000; Gable, y Haidt, 2005); siendo clave la reciprocidad de la gratitud entre profesorado y alumnado por participar en el proceso de enseñanza-aprendizaje, siempre en función de su amor, bondad e inteligencia social como acción benéfica o moral que busca el bienestar pro-social de las personas participantes (Bartlett, y DeSteno, 2006; Nowak, y Roch, 2007), profesoradoalumnado, infiriendo intencionalidad y búsqueda del beneficio compartido y cooperativo como intercambio recíproco guiado por las emociones y el afecto moral (McCullough, Kilpatrick, Emmons, y Larson, 2001; McCullough, Kimeldorf, y Cohen, 2008).

Con este estudio de investigación se pretende comprobar si la acción de mostrar gratitud influye en el rendimiento académico de los estudiantes de una segunda lengua; formulando la siguiente hipótesis: la gratitud recíproca favorece el rendimiento académico de los estudiantes de lenguas. 


\section{Método}

\section{Participantes}

Ciento cuarenta y cinco estudiantes (101 mujeres y 44 hombres) matriculados en cuatro secciones de la asignatura "HSST-1000-Introduction to Spanish" del departamento de Lenguas Modernas y sus Literaturas de Trent University (Canadá), con edades comprendidas entre 19 y 34 años $(N=145, M=22.1 ; S D=3.42)$. Los participantes pertenecían a cuatro secciones diferentes, dos del primer semestre del curso académico 2013-14 y las otras dos al primer semestre del curso académico 2014-15. El Grupo Experimental, GE, se constituyó con dos secciones ( $N=66)$, una de cada año académico; el Grupo Control, GC, también se constituyó de dos secciones $(N=79)$. Los datos descriptivos se muestran en la tabla 1.

\begin{tabular}{|lccccccc|}
\hline \multicolumn{7}{|c|}{ Tabla 1 } & \\
& Estadísticos descriptivos de género y edad & \\
\hline Grupo & Mujer & Hombre & N & Mínimo & Máximo & Media & Desv. típ. \\
\hline & 101 & 44 & 145 & 19 & 34 & 22,09 & 3,424 \\
\hline Control & 55 & 22 & 79 & & & & \\
\hline Experimental & 46 & 20 & 66 & & & & \\
\hline
\end{tabular}

\section{Procedimiento e instrumentos}

Este estudio se realizó en Trent University (Canadá), durante el primer semestre de los cursos académicos 2013-14 y 2014-15, en la asignatura de lengua moderna HSST-1001H-Introduction to Spanish del Department of Modern Languages \& Literatures.

Durante ambos semestres se implementó un programa de gratitud, PG, que consistía en mostrar la gratitud al alumnado por participar y/o asistir a la clase; la gratitud se mostró de forma colectiva y con humor, utilizando técnicas de grupo en feedback y desarrollo personal, con la intención de generar cohesión grupal y mejorar las relaciones interpersonales; el humor y la imaginación fueron claves para crear un proceso simple de bienestar y felicidad, facilitando el sentimiento de gratitud y necesidad de reciprocidad (Fredrickson, y Losada, 2005; Lyubomirsk, y Layous, 2013).

Los participantes tuvieron que realizar dos pruebas objetivas de contenidos teóricoprácticos de la asignatura HSST-1001H-Introduction to Spanish. La primera prueba, $\mathrm{P} 1$, se realizó en la segunda semana de octubre. Después se implementó el Programa de Gratitud al Grupo Experimental. Posteriormente los participantes realizaron la segunda prueba, P2, la cual se realizó durante la tercera semana de noviembre.

Finalmente se procedió a analizar los resultados obtenidos de ambas pruebas (P1 y P2) con el programa estadístico SPSS 20; concretamente se realizó una comparación estadística de las medias, a través de la prueba T para muestras independientes.

\section{Resultados}

Antes de proceder con el análisis estadístico, los resultados obtenidos se muestran en tres diagramas radiales. En el primer diagrama se visualizan los resultados obtenidos de ambas pruebas pudiéndose apreciar una pequeña diferencia entre la P1 y la P2 en ambos grupos; el GE incrementa su rendimiento en la P2 respecto a la P1, mientras que el GC disminuye en la $\mathrm{P} 2$ respecto a la $\mathrm{P} 1$. Los siguientes diagramas radiales muestran los resultados obtenidos por cada grupo, GC y GE, para ambas pruebas, P1 y P2; en ambos se visualizan los resultados anteriores, pero separadamente por 
grupo; así se puede apreciar que existen diferencias entre la prueba P1 y la P2, cuya tendencia en el GC es a disminuir, posiblemente debido a que se incrementa el nivel de dificultad; sin embargo el GE incrementó los resultados.

Por lo tanto, se puede apreciar visualmente que existen diferencias entre los resultados de rendimiento obtenidos entre los grupos experimental y control. En este estudio se analizan estadísticamente los resultados obtenidos en ambas pruebas, por ambos grupos, con la intención de comprobar si las diferencias que se aprecian en los diagramas radiales son significativas.

\section{Gráficas}

Diagramas radiales de los resultados obtenidos en ambas pruebas, P1 y P2, para ambos grupos, GE y GC.

\section{Resultados de P1 Y P2 por GE y GC}

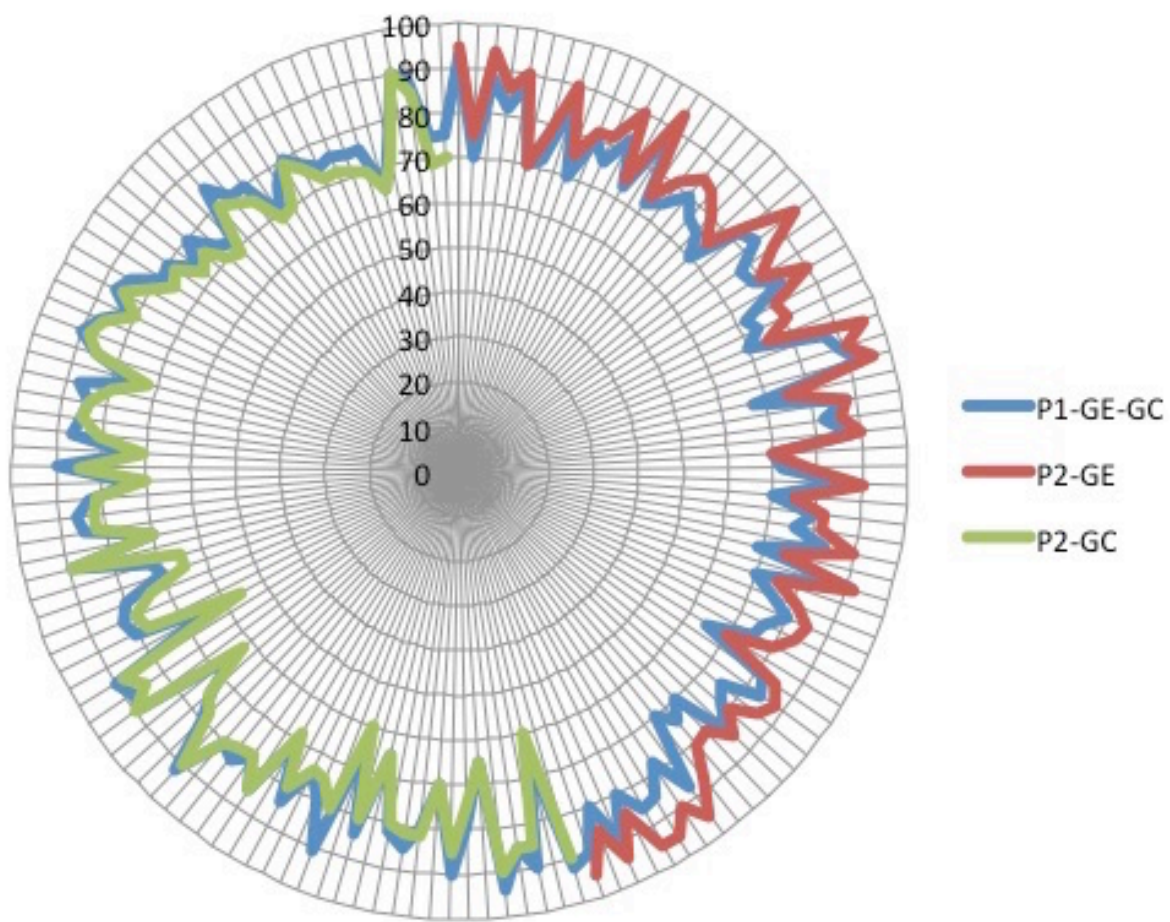

Resultados de P1 Y P2 por GC

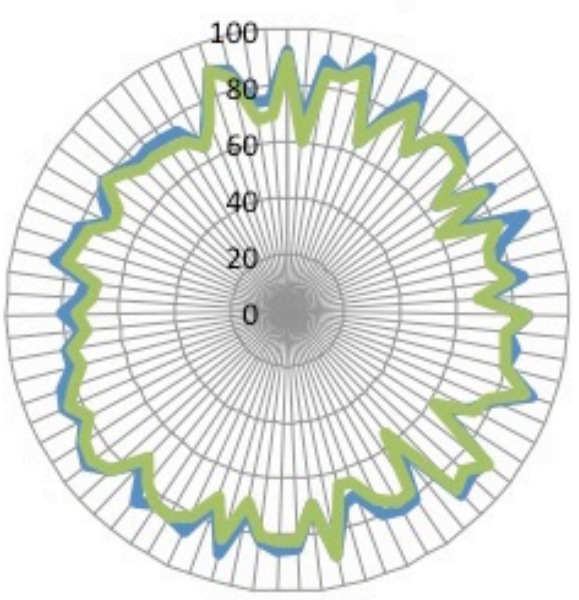

$\longrightarrow$ P1-GC

P2-GC
Resultados de P1 Y P2 por GE

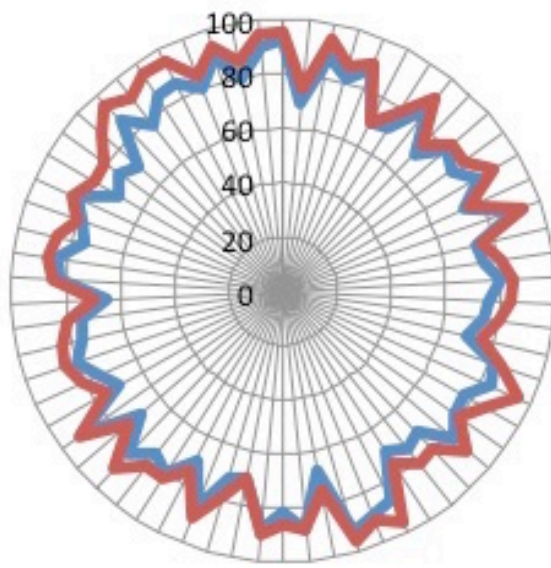

$\longrightarrow$ P1-GE

$\longrightarrow$ P2-GE 
A continuación se procede a analizar estadísticamente los resultados. Primero se procede a realizar la prueba de normalidad de Kolmogorov-Smirnov (tabla 2), siendo la significancia mayor de 0.05 para ambos grupos (P1-GC: $p=.1, \mathrm{P} 1-\mathrm{GE}: p=.169, \mathrm{P} 2-\mathrm{GC}$ : $p=.071$, P2-GE: $p=.092$ ), lo que confirma que los datos provienen de una distribución normal.

\begin{tabular}{|llccc|}
\hline \multicolumn{5}{|c|}{ Tabla 2 } \\
\hline & GRUPO & \multicolumn{3}{c|}{ Shapiro-Wilk } \\
\cline { 3 - 5 } & & Estadístico & Gl & Sig. \\
\hline P1 & Control &, 091 & 79 &, 100 \\
\cline { 2 - 5 } & Experimental &, 100 & 66 &, 169 \\
\hline P2 & Control &, 096 & 79 &, 071 \\
\cline { 2 - 5 } & Experimental &, 101 & 66 &, 092 \\
\hline *. Este es un límite inferior de la significación verdadera. \\
a. Corrección de la significación de Lilliefors. \\
\hline
\end{tabular}

Por lo tanto, procede comparar estadísticamente las medias de los datos obtenidos de ambas pruebas, a través de la prueba T para muestras independientes. A continuación se comprueba el resultado de la prueba de Levene para la igualdad de varianzas, así podemos confirmar que se asumen varianzas iguales para ambas pruebas ( $\mathrm{P}$ 1: $F=.037, p=.847 ; \mathrm{P} 2: F=.932, p=.336)$.

Después se comprueba la prueba $\mathrm{T}$ para la $\mathrm{P} 1$, la cual indica que no existen diferencias significativas entre los resultados obtenidos por los participantes del Grupo Control $(M=79.75, S E=.878)$ y el Grupo Experimental $(M=79.02, S E=0.897)$. Por consiguiente antes de mostrar la gratitud no existían diferencias significativas entre ambos grupos.

Sin embargo la prueba T para la P2 indica que existen diferencias significativas entre los resultados obtenidos por los participantes del Grupo Control $(M=76.96, S E=.936)$ y los del Grupo Experimental, con una potencia alta que explica aproximadamente el $25 \%$ del efecto de la varianza total $(M=84.35, S E=.890, t(143)=5.749, p=.0001$, $r=0.434$ ). También entre los intervalos de confianza para la diferencia no se encuentra el 0, confirmando que el análisis es correcto (Inferior: -9.922, Superior: -4.851). Por lo tanto, se puede afirmar que el análisis estadístico confirma que la gratitud mostrada hacia el alumnado por participar y/o asistir a clase influye positivamente en la calificación de la segunda prueba. Así que la gratitud ha sido significativamente eficiente y positiva en el rendimiento de los participantes del Grupo Experimental. El análisis se muestra con más detalle en las tablas 3 y 4 .

\begin{tabular}{|cccccc|}
\hline \multicolumn{5}{c|}{ Tabla 3 } \\
& \multicolumn{5}{c|}{ Estadísticos de grupo } \\
\hline \multirow{2}{*}{ P1 } & GRUPO & $\mathrm{N}$ & Media & Desviación típ. & Error típ. de la media \\
\cline { 2 - 6 } & Control & 79 & 79,75 & 7,803 &, 878 \\
\hline \multirow{2}{*}{ P2 } & Experimental & 66 & 79,02 & 7,291 &, 897 \\
\cline { 2 - 6 } & Control & 79 & 76,96 & 8,053 &, 936 \\
\hline
\end{tabular}

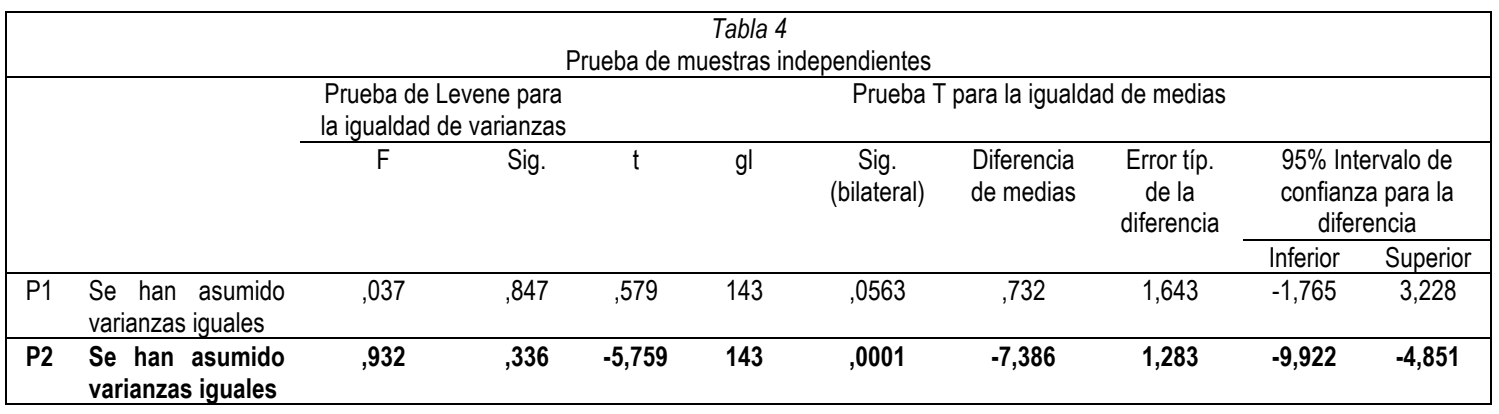




\section{Discusión y conclusiones}

Este estudio demuestra una relación estadísticamente significativa entre gratitud y rendimiento académico en los estudiantes de español como segunda lengua, lo cual permite defender la necesidad de hacer una educación humanística, de carácter social. De los resultados obtenidos se puede afirmar que las personas que sienten agradecimiento mejoran el rendimiento, coincidiendo con investigaciones previamente realizadas en las que se encontraron que existía vinculación entre la gratitud, las emociones y la inteligencia (Goleman, 2006; Fiorini, y Garcia-Ramirez, 2013).

En síntesis, con este estudio se demuestra empíricamente la importancia de la gratitud en la educación, ya que está relacionada con las emociones tanto del alumnado como del profesorado. Además, en una sociedad humanista, las personas que son referentes deben mostrar agradecimiento. La gratitud es un sentimiento que conlleva la sensación de felicidad y bienestar la cual se puede transferir a otras personas tanto del endogrupo como del exogrupo, potenciando las relaciones interpersonales e intrapersonales, lo cual es clave para el éxito de la enseñanza de una segunda lengua. En el aula la gratitud se puede hacer intencional, pero partiendo del profesorado como referente. Generalmente el profesorado espera que el alumnado le agradezca su trabajo, pero la educación es un proceso de enseñanza-aprendizaje en el que todos sus miembros, tanto profesorado como alumnado, son iguales, sólo existe una diferencia provisional de rol (Garcia-Ramirez, 2014).

Este estudio es de carácter exploratorio, por lo que representa el inicio de una línea de investigación para comprender qué aspectos son decisivos para mejorar el rendimiento no sólo en el aula de lenguas sino en la educación en general.

Entre las limitaciones de este estudio se encuentran los factores de riesgo psicosocial que afectan al humor tanto al alumnado como al profesorado, sobre todo las exigencias psicológicas, estima y apoyo social.

Concluyendo, para que el alumnado muestre un comportamiento de gratitud es necesario que el profesorado posibilite la reciprocidad y la intencionalidad en el proceso de enseñanza-aprendizaje.

\section{Referencias}

Bartlett, M., y DeSteno, D. (2006). Gratitude and Prosocial Behavior Helping when it Costs You. Psychological Science, 17(4), 319-325. http://dx.doi.org/10.1111/j.1467-9280.2006.01705.x

Brülde, B. (2007). Happiness theories of the good life. Journal of Happiness Studies, 8(1), 15-49. http://dx.doi.org/10.1007/s10902-006-9003-8

Emmons, R.A. (2008). Thanks! How the new science of gratitude can make you happier. New York: Houghton-Mifflin. ISBN 13: 9780547085739.

Emmons, R.A., y Crumpler, C.A. (2000). Gratitude as a human strength: Appraising the evidence. Journal of Social and Clinical Psychology, 19(1), 56-69. http://dx.doi.org/10.1521/jscp.2000.19.1.56 
Emmons, R.A., y McCullough, M.E. (2003). Counting blessings versus burdens: an experimental investigation of gratitude and subjective well-being in daily life. Journal of personality and social psychology, 84(2), 377. http://dx.doi.org/10.1037/0022-3514.84.2.377

Fiorini, M.; García-Ramírez, JM. (2013). Cap. 5: Técnicas de grupo y creatividad aplicadas en el ámbito universitario,117-147. En M.D. Villena Martínez, MD.; A. Muñoz García, A. (2013). Recursos para la tutoría en el aula universitaria. Granada: Editorial Universidad de Granada. ISBN 13: 9788433855626.

Fredrickson, B.L., y Losada, M.F. (2005). Positive affect and the complex dynamics of human flourishing. American Psychologist, 60(7), 678-686. http://dx.doi.org/10.1037/0003-066X.60.7.678

Gable, S.L., y Haidt, J. (2005). What (and why) is positive psychology? Review of general psychology, 9(2), 103-110. http://dx.doi.org/10.1037/1089-2680.9.2.103

Garcia-Ramirez, J.M. (2014). Selección de indicadores para la evaluación de la excelencia docente en la Universidad de Trent (Canadá). Granada: Universidad de Granada, 2014. http://hdl.handle.net/10481/30350

Goleman, D. (2006). Inteligencia Social. La nueva ciencia para mejorar las relaciones humanas. México D.F.: Planeta. ISBN 10: 9703705626.

Lyubomirsky, S., y Layous, K. (2013). How do simple positive activities increase wellbeing? Current Directions in Psychological Science, 22(1), 57-62. http://dx.doi.org/10.1177/0963721412469809

McCullough, M.E., Emmons, R.A., y Tsang, J.A. (2002). The grateful disposition: a conceptual and empirical topography. Journal of personality and social psychology, 82(1), 112-127. http://dx.doi.org/10.1037/0022-3514.82.1.112

McCullough, ME., Kilpatrick, S. D., Emmons, R.A., y Larson, D.B. (2001). Is gratitude a moral affect? Psychological bulletin, 127(2), 249-266. http://dx.doi.org/10.1037/0033-2909.127.2.249

McCullough, M.E., Kimeldorf, M.B., y Cohen, A.D. (2008). An Adaptation for Altruism The Social Causes, Social Effects, and Social Evolution of Gratitude. Current Directions in Psychological Science, 17(4), 281-285. http://dx.doi.org/10.1111/j.1467-8721.2008.00590.x

McCullough, M.E., Tsang, J.A., y Emmons, R.A. (2004). Gratitude in intermediate affective terrain: links of grateful moods to individual differences and daily emotional experience. Journal of Personality and Social Psychology, 86(2), 295-309. http://dx.doi.org/10.1037/0022-3514.86.2.295

Nowak, M.A., y Roch, S. (2007). Upstream reciprocity and the evolution of gratitude. Proceedings of the Royal Society B: Biological Sciences, 274(1610), 605-610. http://dx.doi.org/10.1098/rspb.2006.0125

Peterson, C., Park, N., y Seligman, M.E. (2005). Orientations to happiness and life satisfaction: The full life versus the empty life. Journal of happiness studies, 6(1), 25-41. http://dx.doi.org/10.1007/s10902-004-1278-z 
Peterson, C., Ruch, W., Beermann, U., Park, N., y Seligman, M.E. (2007). Strengths of character, orientations to happiness, and life satisfaction. Journal of Positive Psychology, 2(3), 149-156. http://dx.doi.org/10.1080/17439760701228938

Sheldon, K.M., y Lyubomirsky, S. (2006). How to increase and sustain positive emotion: The effects of expressing gratitude and visualizing best possible selves. The Journal of Positive Psychology, 1(2), 73-82. http://dx.doi.org/10.1080/17439760500510676

Tsang, J.A. (2006). The effects of helper intention on gratitude and indebtedness. Motivation and Emotion, 30(3), 198-204. http://dx.doi.org/10.1007/s11031-0069031-z

Watkins, P., Scheer, J., Ovnicek, M., y Kolts, R. (2006). The debt of gratitude: Dissociating gratitude and indebtedness. Cognition y Emotion, 20(2), 217-241. http://dx.doi.org/10.1080/02699930500172291

Wood, A.M., Froh, J.J., y Geraghty, A.W. (2010). Gratitude and well-being: A review and theoretical integration. Clinical psychology review, 30(7), 890-905. http://dx.doi.org/10.1016/j. 